\title{
Asymptotic expansion of the full nonlocal solidification problem
}

\author{
Wim van Saarloos and John D. Weeks \\ AT\&T Bell Laboratories, Murray Hill, New Jersey 07974-2070
}

\author{
Gabriel Kotliar \\ Department of Physics, ${ }^{*}$ Massachusetts Institute of Technology, Cambridge, Massachusetts 02139 \\ and AT\& T Bell Laboratories, Murray Hill, New Jersey 07974-2070
}

(Received 22 October 1986)

\begin{abstract}
We analyze the shape $z(x)$ of two-dimensional needle crystals far away from the tip and find that in general the deviation $\Delta z$ away from the Ivantsov solution has an asymptotic behavior of the form $\Delta z \sim x^{-\alpha}$, with $\alpha$ a noninteger exponent. For the asymptotic behavior, the regime where the Péclet number $p$ is less than $\frac{1}{2}$ and the one where $p$ is larger than $\frac{1}{2}$ are distinct. For $p>\frac{1}{2}$, the exponent is calculated explicitly, while for $p<\frac{1}{2}$, we present numerical evidence for the existence of the exponent $\alpha$. These results differ from those used in earlier numerical and analytical studies of twodimensional dendritic growth.
\end{abstract}

The analysis of the steady-state equations of the nonlocal solidification problem ${ }^{1-4}$ has recently attracted a lot of attention motivated by the possible connections between the solvability of the steady-state equations and the dynamical velocity selection. ${ }^{5,6}$

An asymptotic analysis of the symmetric model in two dimensions was performed by Kessler et al. ${ }^{2}$ who concluded that the shape of the solidification front moving in the $z$ direction with velocity $V$ is far away from the tip given by $z(x)=-x^{2} / 2 p+c+a /|x|$. Here $p$ is the Péclet number of the Ivantsov solution, and all lengths are measured in units of the diffusion length $2 D / V$. The coefficient $a$, according to Kessler et al., ${ }^{2}$ is a Péclet-numberdependent constant whose value does not depend on the details of the shape in the tip region; the constant $c$ is arbitrary and of no physical significance, since it can be made to vanish by translating the whole shape in the $z$ direction by an amount $-c .^{7}$ In this note we reconsider this problem by investigating what asymptotic behavior is allowed as a function of $p$ in the integral equation obtained by linearizing the full equation about the Ivantsov solution. For $p<1 / 2$ we conclude on the basis of analytical as well as numerical results that

$$
z(x)=-\frac{1}{2 p} x^{2}+c+\frac{a}{x^{\alpha}}, \alpha(p)>1, p<\frac{1}{2} .
$$

The coefficient $a$ depends on the complete shape of the needle crystal and cannot be determined only from the tail; moreover, it appears that the exponent $\alpha$ cannot be determined solely from the asymptotic large- $x$ behavior of the linearized equation. In numerical solutions of the full equation, $\alpha$ is found to vary with $p$. For $p>\frac{1}{2}$ we find that in general it is possible to have

$z(x)=-\frac{1}{2 p} x^{2}+b|x|^{\beta}+c,-1<\beta(p)<1, \quad p>\frac{1}{2}$, where the coefficient $b$ is arbitrary within the asymptotic analysis. The value of $b$ can therefore only be determined by matching the tail expansion (2) to the profile in the tip region. Clearly, the structure of the asymptotic solution in the tails of the needle is rather different from what was claimed before. ${ }^{2}$

The issue of whether the steady-state equations have solutions for a continuous range of values of $d_{0}$ or for a discrete set of values is still a subject of some debate. We do not intend to address this issue here, but only note that the dominant term in an "outer expansion" for the large- $x$ regime, from which the results (1) and (2) also follow, will be interpreted physically and discussed in the light of this question elsewhere. ${ }^{8}$ Our results in this paper are valid for any steady-state solution, if one exists, or for any generalized solution studied in the numerical approaches, ${ }^{1,2}$ for which $z^{\prime} \equiv d z / d x \neq 0$ at the tip.

The two-dimensional steady-state equations for the symmetric two-sided model of dendritic growth are given by ${ }^{9,10}$

$$
D \nabla^{2} T+V \frac{\partial T}{\partial z}=0,
$$

subject to the boundary conditions ([ ] denotes the discontinuity across the boundary at the solid-liquid interface)

$$
\begin{aligned}
& D\left[\frac{\partial T}{\partial n}\right]=L \frac{V_{n}}{C_{p}}, \\
& T=T_{M}\left(1-\bar{d}_{0} \kappa\right) .
\end{aligned}
$$

Here $\kappa$ denotes the curvature, $D$ the diffusion constant, $C_{p}$ the specific heat, $L$ the latent heat, $\bar{d}_{0}$ a capillary length, $T_{M}$ the bulk melting temperature, $V$ the propagation velocity along the $z$ direction, and $V_{n}$ the normal component of the velocity. Measuring all temperatures in units of $L / C_{p}$ and the lengths in units of the diffusion length $2 D / V$, the steady-state problem reduces to the integro-differential equation 9,10 


$$
\begin{aligned}
& \Delta+ \frac{d_{0} z^{\prime \prime}}{\left[1+z^{\prime}(x)^{2}\right]^{3 / 2}} \\
&=\int_{-\infty}^{\infty} \frac{d x_{1}}{\pi} e^{\left[z\left(x_{1}\right)-z(x)\right]} K_{0} \\
& \quad \quad \times K_{0}\left(\left\{\left(x-x_{1}\right)^{2}+\left[z(x)-z\left(x_{1}\right)\right]^{2}\right\}^{1 / 2}\right) .
\end{aligned}
$$

where $\Delta$ is the dimensionless undercooling,
$d_{0}=T_{m} C_{p} \bar{d}_{0} V / 2 D L$, and $K_{0}$ is the zeroth-order modified Bessel function.

We will now study the asymptotic regime $x \rightarrow \infty$ for any possible steady-state solution of Eq. (6). Since Eq. (6) admits Ivantsov solutions $z=-(1 / 2 p) x^{2}$ for $d_{0}=0$, we write $z=-(1 / 2 p) x^{2}+\Delta z$ and linearize in $\Delta z$ (we will come back to the validity of the linearization later). For the asymptotic large $x$ behavior of (6) we then get, using $K_{0}^{\prime}=-K_{1}$,

$$
\begin{aligned}
-\frac{d_{0} p^{2}}{x^{3}}= & \int_{0}^{\infty} \frac{d x_{1}}{\pi} e^{\left(x^{2}-x_{1}^{2}\right) / 2 p}\left(-K_{0}\left(r_{-}\right)+K_{1}\left(r_{-}\right) \frac{x^{2}-x_{1}^{2}}{2 p r_{-}}\right)\left[\Delta z(x)-\Delta z\left(x_{1}\right)\right] \\
& +\int_{0}^{\infty} \frac{d x_{1}}{\pi} e^{\left(x^{2}-x_{1}^{2}\right) / 2 p}\left(-K_{0}\left(r_{+}\right)+K_{1}\left(r_{+}\right) \frac{x^{2}-x_{1}^{2}}{2 p r_{+}}\right)\left[\Delta z(x)-\Delta z\left(x_{1}\right)\right],
\end{aligned}
$$

with

$$
\begin{aligned}
& r_{-}^{2} \equiv\left(x-x_{1}\right)^{2}+\frac{1}{4 p^{2}}\left(x^{2}-x_{1}^{2}\right)^{2}, \\
& r_{+}^{2} \equiv\left(x+x_{1}\right)^{2}+\frac{1}{4 p^{2}}\left(x^{2}-x_{1}^{2}\right)^{2} .
\end{aligned}
$$

The main contribution to Eq. (7) comes from the region $[0, x]$; more precisely, consider, e.g., ${ }^{11} x_{1}$ in the range $\left[0, x-x^{-1 / 2}\right]$ in the first integral and in the range $\left[0, x-x^{1 / 4}\right]$ in the second integral, so that we can use the asymptotic expressions

$$
\begin{aligned}
& K_{0}(r) \cong\left(\frac{\pi}{2}\right)^{1 / 2} \frac{e^{-r}}{\sqrt{r}}\left(1-\frac{1}{8 r}\right), \\
& K_{1}(r) \cong-\left(\frac{\pi}{2}\right)^{1 / 2} \frac{e^{-r}}{\sqrt{r}}\left(1+\frac{3}{8 r}\right), \\
& r_{-} \cong \frac{1}{2 p}\left(x^{2}-x_{1}^{2}\right)+\frac{\left(x-x_{1}\right) p}{\left(x+x_{1}\right)} \\
& r_{+} \cong \frac{1}{2 p}\left(x^{2}-x_{1}^{2}\right)+\frac{p\left(x+x_{1}\right)}{x-x_{1}}
\end{aligned}
$$

Changing variables $x_{1}=x(1-v) /(1+v)$ in the first integral and $x_{1}=x(v-1) /(1+v)$ in the second we arrive at $^{12}$

$$
\begin{aligned}
-\frac{d_{0} p^{2}}{x^{3}}=\frac{1}{2 \sqrt{\pi}} \int_{0}^{\infty} & d v \frac{(1+v)}{\sqrt{v}} \frac{e^{-p v}}{x^{2}} p^{3 / 2}\left(p-\frac{1}{2 v}\right) \\
& \times\left[\Delta z\left(\frac{x(1-v)}{1+v}\right)-\Delta z(x)\right] .
\end{aligned}
$$

Two cases have to be considered separately in analyzing Eq. (10): the one where the dominant term in $\Delta z$ for large $x$ gives a contribution to the integral of order $x^{-3}$, and the one where the leading term in $\Delta z$ makes the integral vanish, so that a subdominant term gives a contribution of order $x^{-3}$. We start by analyzing the first case.

Since $\Delta z(x)$ is assumed to be an even function of $x[\Delta z(x)=\Delta z(-x)]$, if $\Delta z(x)$ decays as $1 / x^{\alpha}$, this introduces a singularity ${ }^{13} 1 /|1-v|^{\alpha}$ in Eq. (10). We show later that $\alpha$ less than 1 (including negative values of $\alpha$ ) is possible only in the second case, in which the dominant term in $\Delta z$ makes the integral vanish. Since it will turn out that $\Delta z$ has to be integrable, we restrict the analysis here to $\alpha>1$. In this case, the singularity $1 /|1-v|^{\alpha}$ is nonintegrable; however, for $v$ close to $1, x(1-v)$ is not necessarily large and it is incorrect to substitute the asymptotic behavior of $\Delta z$ in (10). This suggests that all parts of the profile, including the tip region, contribute to the integral in (10). To make this more precise, it is convenient to split up the $v$ integral into two parts, with $|1-v|<\epsilon x^{-1 / 2}$ and $|1-v|>\epsilon x^{-1 / 2}$. Since $x(1-v)$ is large in the latter range, we can approximate $\Delta z(x)$ there by the asymptotic behavior $\Delta z \approx a / x^{\alpha}$. The integral over this range then behaves as

$$
\frac{a p^{3 / 2}}{2 \sqrt{\pi} x^{2+\alpha}} \int_{|1-v|>\epsilon x^{-1 / 2}} d v \frac{1+v}{\sqrt{v}} e^{-p v}\left[p-\frac{1}{2 v}\right]\left[\left[\frac{1+v}{1-v}\right]^{\alpha}-1\right]=O\left(x^{-(5+\alpha) / 2}\right), \quad \alpha>1,
$$

which decays faster than the $x^{-3}$ term on the left-hand side $e^{14}$ of Eq. (10). Hence on comparing terms of order $x^{-3}$ in (8), only the integral over the region $|1-v|<\epsilon x^{-1 / 2}$ has to be retained. After a change of variables $v=1+2 w / x$, we then get $^{15}$ for large $x$

$$
-\frac{d_{0} p^{2}}{x^{3}}=\frac{p^{3 / 2}}{\pi^{1 / 2} x^{3}} \int_{-\epsilon x^{1 / 2} / 2}^{\epsilon x^{1 / 2} / 2} d w \frac{2+2 w / x}{(1+2 w / x)^{1 / 2}} e^{-p(1+2 w / x)}\left(p-\frac{1}{2+4 w / x}\right) \Delta z(w /(1+w / x)) .
$$


For large $x$, the terms $w / x$ are negligible in the range of integration, and the integral approaches a well-defined limit. We then get

$$
-d_{0} p^{1 / 2}=\frac{2}{\pi^{1 / 2}}\left(p-\frac{1}{2}\right) e^{-p} \int_{-\infty}^{+\infty} d w \Delta z(w) .
$$

This equation confirms that $\Delta z(x)$ has to be integrable, but otherwise does not determine the functional form of the asymptotic behavior of $\Delta z$. Clearly, if we assume $\Delta z \approx a / x^{\alpha}$ as in Eq. (1), the determination of the coefficient $a$ depends on the solution of the problem close to the tip, and cannot be derived solely from asymptotic considerations as claimed in Ref. 2. We have solved Eq. (6) numerically, and observed indeed such power-law behavior (see Fig. 1); $\alpha$ is in general found to be noninteger, significantly larger than 1 for small $p$, and independent of $d_{0}$. Thus, the numerical solutions appear to confirm the asymptotic from (1), although on the basis of the numerical results we cannot rule out a small $d_{0}$ dependence or asymptotic behavior of the form $x^{-\alpha} \ln x$ (in fact, the slight curvature in the data of Fig. 1 for $p=0.25$ might be an indication of the presence of such type of terms). Note also that according to (13) $\int d w \Delta z(w)$ diverges as $p \rightarrow \frac{1}{2}$, suggesting that $\alpha \rightarrow 1$ in this limit. Indeed, for increasing Péclet numbers, the slope in Fig. 1 decreases.

If one makes the Ansatz $\Delta z \sim 1 / x$, both the term in Eq. (11) and the one in Eq. (12) become of order $x^{-3} \ln x$. Since we have not proven that these two terms cannot cancel one another, we have, strictly speaking, not excluded the possibility $\alpha=1$. We have found no indications that such cancellations occur, nor observed $\Delta z \sim 1 / x$ in our numerical solutions for $p<\frac{1}{2}$.

The above considerations, do not apply if the dominant asymptotic behavior of $\Delta z$ gives a vanishing contribution to the right-hand side of Eq. (10); i.e., is a solution of the

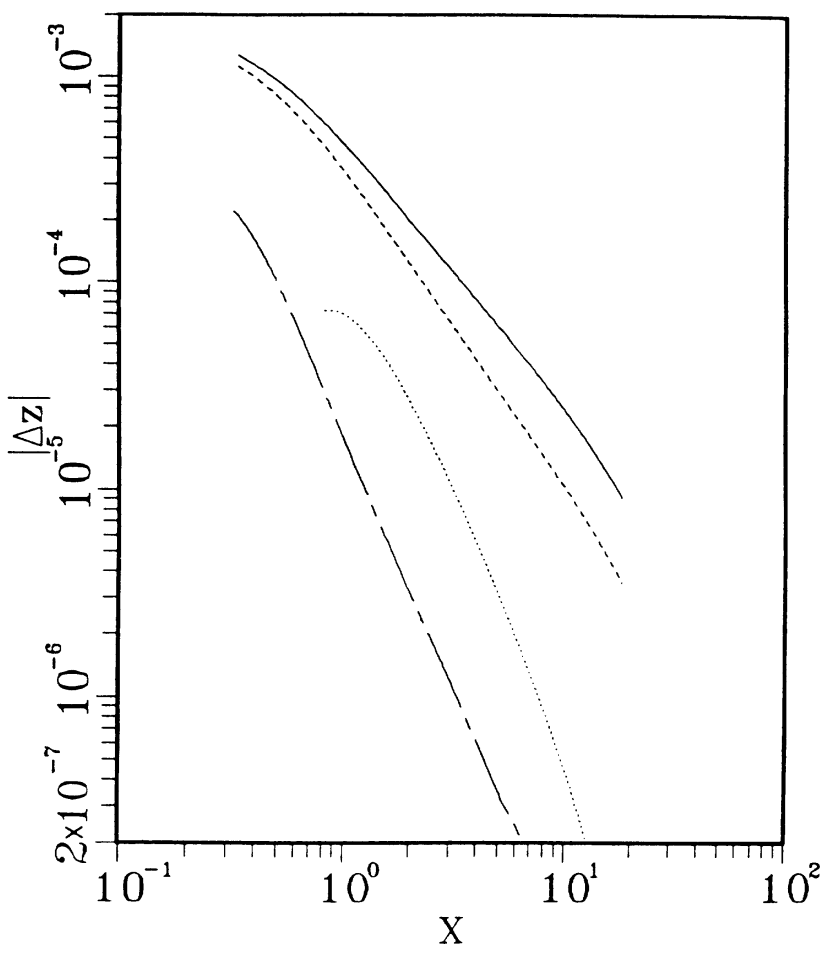

FIG. 1. Log-log plot of $|\Delta z-c|$ vs $x$ obtained from numerical solutions with $d_{0}=0.0002$ at $p=0.475$ (solid line, $\alpha \approx 1.25$ ), $p=0.45$ (short dashes, $\alpha \approx 1.45$ ), $p=0.25$ (dots, $\alpha \approx 2.5$ ), and $P=0.1$ (long dashes, $\alpha \approx 2.5$ ).

homogeneous equation obtained by setting the left-hand side of Eq. (10) equal to zero. We will see that this is possible for $p>\frac{1}{2}$. Upon substituting $\Delta z \sim x^{\beta}$ with $\beta>-1$ and rewriting the integral from 1 to $\infty$ with the aid of the transformation $v \rightarrow 1 / v$, we get

$$
\frac{x^{\beta-2} p^{3 / 2}}{2 \sqrt{\pi}} \int_{0}^{1} d v \frac{1+v}{\sqrt{v}}\left[e^{-p v}\left[p-\frac{1}{2 v}\right]+e^{-p / v}\left(\frac{p}{v^{2}}-\frac{1}{2 v}\right]\right]\left[\left[\frac{1-v}{1+v}\right)^{\beta}-1\right]=0
$$

It is easy to see that the first term between square brackets changes sign for $p>\frac{1}{2}$. Since the second term between square brackets weights different parts of the interval $[0,1]$ differently depending on $\beta$, the integral will vanish for some value of $\beta$, implying that Eq. (14) always has a solution for $p>\frac{1}{2}$. This justifies Eq. (2), with the exponent $\beta$ given by Eq. (14). A plot of $\beta(p)$ obtained by solving the latter equation numerically is shown in Fig. 2 . Note that $\beta \rightarrow-1$ as $p \rightarrow \frac{1}{2}$, so that we expect the exponents $\alpha$ and $\beta$ to be continuous at $p=\frac{1}{2}$, at which point presumably $z \sim 1 / x$ or $z \sim x^{-1} \ln x$. In Fig. 3 we plot $\Delta z$ as obtained from a numerical solution of Eq. (6) for $p=1$ on a log-log scale. The behavior is indeed consistent with Eq. (2), although the effective exponent is still slowly decreasing. By extending our solution to large values of $x$

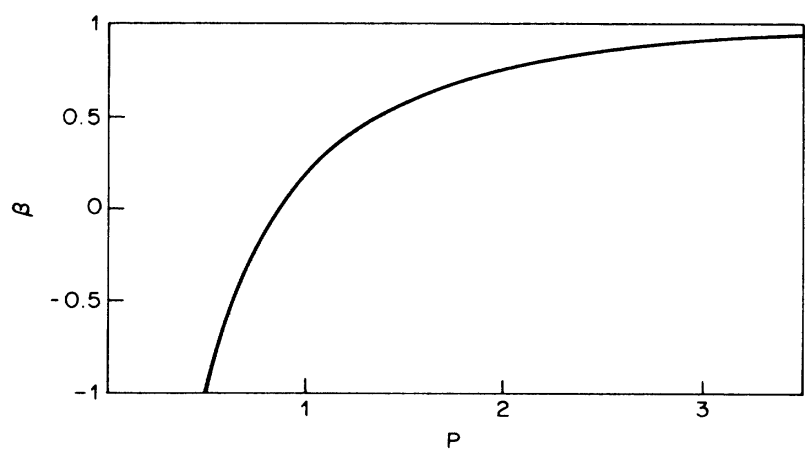

FIG. 2. Exponent $\beta$ as a function of the Ivantsov Péclet number $p$, as determined by Eq. (14). 


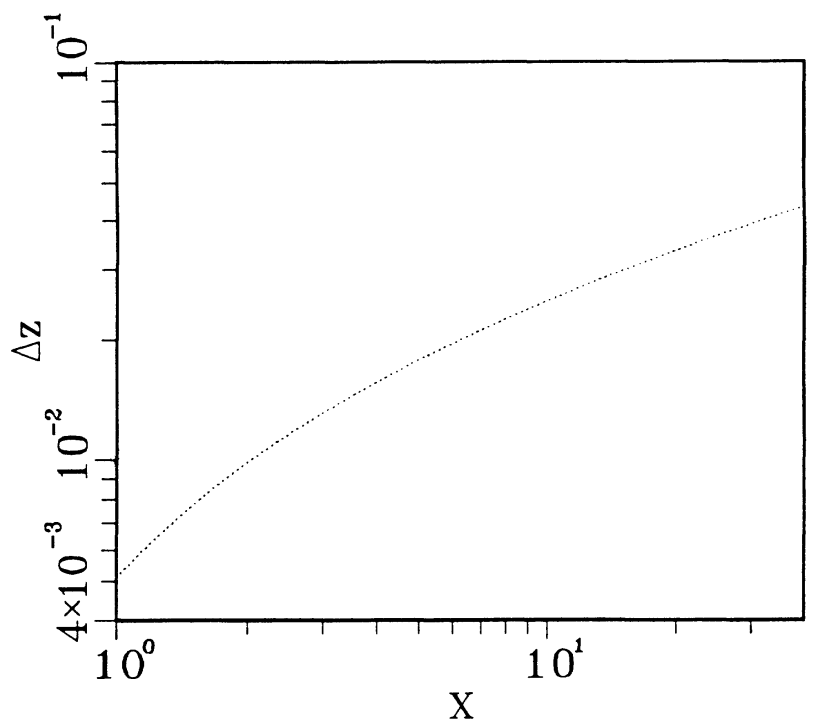

FIG. 3. Log-log plot of $\Delta z$ vs for $\Delta=0.76(p \approx 1)$. The behavior is consistent with Eq. (2), with the effective exponent $\beta$ slowly decreasing towards its predicted value of about 0.2 .

(up to 4000), we have checked that the effective exponent does continue to approach the value of about 0.2 predicted by Eq. (14). We note that the data of Fig. 5 of Meiron ${ }^{1}$ for $p=1$ also appear to be consistent with Eq. (2), with an effective exponent between $\frac{1}{3}$ and $\frac{1}{2}$ for $x \approx 10$.

When $\beta>0, \Delta z$ diverges; in view of the exponential term $\exp \left[z\left(x_{1}\right)-z(x)\right]$ in the integrand in Eq. (6), one might at first sight conclude that the linearization leading to Eqs. (10) and (14) is inconsistent with this result. However, on closer inspection we find that this is not the case. For, in the regime where the asymptotic expressions (9) are valid, the dominant term $\exp (-r)$ of the Bessel function partly cancels the exponentially large term $\exp \left[z\left(x_{1}\right)-z(x)\right]$, and one gets for $x_{1}$ in the regime considered

$$
e^{z\left(x_{1}\right)-z(x)} e^{-r} \cong \exp \left(\frac{-\left(x-x_{1}\right)^{2}}{2\left|z\left(x_{1}\right)-z(x)\right|}\right) .
$$

Since $z(x)$ is approximately parabolic, the exponent on the right-hand side does not diverge for large $x$. Moreover, Eq. (15) also shows that linearization in $\Delta z$ in fact amounts to an expansion in $\Delta z / z_{i v}=-2 p \Delta z / x^{2}$, which is always small for large $x$. This is the origin of the term $x^{-2}$ in Eq. (10), and implies that Eq. (14) also holds for positive $\beta$.

In the above analysis the dominant term in the integral for $p>\frac{1}{2}$ comes from the tail region, while for $p<\frac{1}{2}$ all parts of the profile contribute to the asymptotic behavior. This, together with the appearance of the factor $p-\frac{1}{2}$ in Eq. (13), suggests that there will be slow transients near $p=\frac{1}{2}$, and a more careful analysis is called for in this limit. As stated before, at $p=\frac{1}{2}$, we expect $\Delta z \sim 1 / x$ with possibly logarithmic corrections.

We also note that the asymptotic behavior is only given by Eqs. (1) and (2) in two dimensions in the absence of interface kinetics. Kinetic undercooling would give rise to a term proportional to $V_{n}=V \cos \theta \sim 1 / x$ on the left-hand side of Eq. (10), and would obviously result in $\Delta z \approx d x+e x^{\beta}+\cdots$. We similarly expect a term linear in $x$ in three dimensions, since there $\kappa$ falls off as $1 / x$.

For completeness we finally show that contributions from other regions of integration in Eq. (7) are asymptotically negligible. Over the interval $\left[x^{-1 / 2}+x, \infty\right]$, $(1 / 2 p)\left(x^{2}-x_{1}^{2}\right)-r_{ \pm} \leq\left(x-x_{1}\right)(x / p)$ and the integrand in Eq. (7) decays exponentially fast. The contribution of the interval $\left[-x^{-1 / 2}+x, x+x^{-1 / 2}\right]$, to the first integral in Eq. (7) is estimated by writing $\Delta z(x)-\Delta z\left(x_{1}\right)$ $\approx \Delta z^{\prime}(x)\left(x-x_{1}\right)$; the integral, which is convergent in spite of the weak singularity of the integrand, then falls off as $(1 / \sqrt{x})^{2}$, and since $\Delta z^{\prime}(x) \sim 1 / x^{\alpha+1}$ the overall contribution is at least ${ }^{16} O\left(1 / x^{\alpha+2}\right)$. Similar considerations show that the contributions of $\left[x-x^{1 / 4}, x+x^{1 / 4}\right]$ to the second integral in Eq. (7) is at least $O\left(1 / x^{2+\alpha}\right)$. Since we found $\alpha>1$, these terms are indeed negligible.

We are grateful to Harvey Segur for his many pertinent comments on an earlier version of the manuscript. G.K. was supported by the National Science Foundation (NSF) under Grant No. DMR-84-18718.
*Present and permanent address.

${ }^{1}$ D. Meiron, Phys. Rev. A 33, 2704 (1986).

${ }^{2}$ D. Kessler, J. Koplik, and H. Levine, Phys. Rev. A 33, 3352 (1986).

${ }^{3}$ A. Barbieri, D. C. Hong, and J. S. Langer, Phys. Rev. A 35, 1802 (1987).

${ }^{4}$ B. Caroli, C. Caroli, B. Roulet, and J. S. Langer, Phys. Rev. A 33, 442 (1986).

${ }^{5}$ D. Kessler, J. Koplik, and H. Levine, Phys. Rev. A 30, 3161 (1984).

${ }^{6}$ E. Ben-Jacob, N. Goldenfeld, G. Kotliar, and J. S. Langer, Phys. Rev. Lett. 53, 2110 (1984).

${ }^{7}$ Note that different conventions are used in Refs. 1 and 2. Meiron puts $z(x=0)=0$, in which case $c \neq 0$, while Kessler et al. use the freedom to choose $z(x=0)$ to make $c$ vanish.
${ }^{8}$ W. van Saarloos and J. D. Weeks (unpublished).

${ }^{9}$ J. S. Langer, Rev. Mod. Phys. 52, 1 (1980).

${ }^{10}$ G. E. Nash, Naval Research Laboratory Reports No. 7679 and No. 7680, 1974 (unpublished).

${ }^{11}$ In general, a range of values of $\gamma$ is allowed such that for $x_{1}$ in the interval $\left[0, x-x^{\gamma}\right]$, one has $r \gg 1$ and such that the second terms in $r_{+}$and $r_{-}$are much larger than the first ones.

${ }^{12}$ As discussed in Ref. 8, the steps leading to Eq. (10) have a clear physical interpretation, and Eq. (10) is the linearized version of an integro-differential equation that admits a continuous family of solution for $d_{0}>0$.

13Reference 2 assumes $\quad \alpha=1, \quad \Delta z(x(1-v) /(1+v))$ $=(1+v) /[(1-v) x]$ and takes the principal value of the integral. This is inconsistent with the fact that $\Delta z$ is assumed to 
be an even function of $x$.

${ }^{14}$ When $\Delta z \sim 1 / x$, the integral is of order $x^{-3} \ln x$.

${ }^{15} \mathrm{We}$ are assuming here that the freedom to choose $\Delta z(x=0)$ has been used to make $\Delta z$ vanish as $x \rightarrow \infty$ [c=0 in Eq. (1)], so that the term $\Delta z(x)$ in (10) does not contribute.

${ }^{16}$ Note that these crude estimates suggest that these terms are only negligible if $\alpha>1$, as found numerically. In fact, however, one can show that the leading contribution of the integrals vanishes because of symmetric integration about $x$ and so the contributions of the region $x \approx x_{1}$ to Eq. (7) is smaller than $O\left(1 / x^{4}\right)$. The analysis of Ref. 8 suggests that the terms considered here are in fact $O\left(x^{-(3+\alpha)}\right)$. 